\title{
Foreign body granuloma in the anterior abdominal wall mimicking an acute appendicular lump and induced by a translocated copper-T intrauterine contraceptive device: a case report
}

\author{
Maulana Mohammed Ansari ${ }^{1 *}$, Syed Hasan Harris ${ }^{1}$, Shahla Haleem ${ }^{2}$, \\ Rehan Fareed $^{1}$ and Mohammed Feroz Khan ${ }^{1}$
}

Addresses: ${ }^{1}$ Department of Surgery, Jawaharlal Nehru, Medical College Hospital, A.M.U., Aligarh, U.P., India and ${ }^{2}$ Department of Anaesthesiology, Jawaharlal Nehru, Medical College Hospital, A.M.U., Aligarh, U.P., India

Email: MMA* - mma_amu@yahoo.com; SHH - hasanharris@yahoo.com; SH - shahlahaleem@yahoo.co.in; RF - rehan.fareed@gmail.com; MFK - firoz_alg99@yahoo.co.in

* Corresponding author

\begin{abstract}
Introduction: Intrauterine contraceptive devices may at times perforate and migrate to adjacent organs. Such uterine perforation usually passes unnoticed with development of potentially serious complications.

Case presentation: A 25-year-old woman of North Indian origin presented with an acute tender lump in the right iliac fossa. The lump was initially thought to be an appendicular lump and treated conservatively. Resolution of the lump was incomplete. On exploratory laparotomy, a hard suspicious mass was found in the anterior abdominal wall of the right iliac fossa. Wide excision and bisection of the mass revealed a copper-T embedded inside. Examination of the uterus did not show any evidence of perforation. The next day, the patient gave a history of past copper-T Intrauterine contraceptive device insertion.
\end{abstract}

Conclusions: Copper-T insertion is one of the simplest contraceptive methods but its neglect with inadequate follow-up may lead to uterine perforation and extra-uterine migration. Regular self-examination for the "threads" supplemented with abdominal X-ray and/or ultrasound in the follow-up may detect copper-T migration early. To the best of our knowledge, this is the first report of intrauterine contraceptive device migration to the anterior abdominal wall of the right iliac fossa. 


\section{Introduction}

Increased patient acceptance of intrauterine contraceptive devices (IUCD), especially copper- $T$, without proper follow-up is associated with many early and late complications, including perforation and migration into adjacent structures in $1 / 350$ to $1 / 2500$ cases [1]. Migration of IUCDs into the urinary bladder, rectum, colon, peritoneum, omentum, appendix, wall of the iliac vein and ovary has been reported [2]. Herein we report the first case of IUCD migration to the anterior abdominal wall in the right iliac fossa (RIF) with foreign body granuloma formation, mimicking an acute appendicular lump.

\section{Case presentation}

A 25-year-old woman was referred to us with a 5 day history of moderate localized pain in her right lower abdomen that was not radiating to any other site and was not associated with nausea or vomiting. The patient had mild pyrexia (temperature $99.4^{\circ} \mathrm{F}$ ). On examination of her abdomen, a well-defined mildly tender localized fixed lump $7 \times 5 \mathrm{~cm}$ in size was found in the right iliac fossa. The hemogram showed a total leukocyte count of $11,000 / \mathrm{mm}^{3}$, with $60 \%$ polymorphonucleocytes. Ultrasonography (USG) of her abdomen revealed an oval-shaped abdominal mass in the right iliac fossa, suggestive of an appendicular lump.

The patient was put on the Ochsner-Sherren regimen. However, recovery was found to be slow and incomplete, and a smaller non-tender lump $5 \times 5 \mathrm{~cm}$ in size was still present at the end of 4 weeks. Repeat USG was suggestive of an unresolved appendicular lump.

On exploratory laparotomy through a lower midline incision, a hard mass lesion was found on the inner side

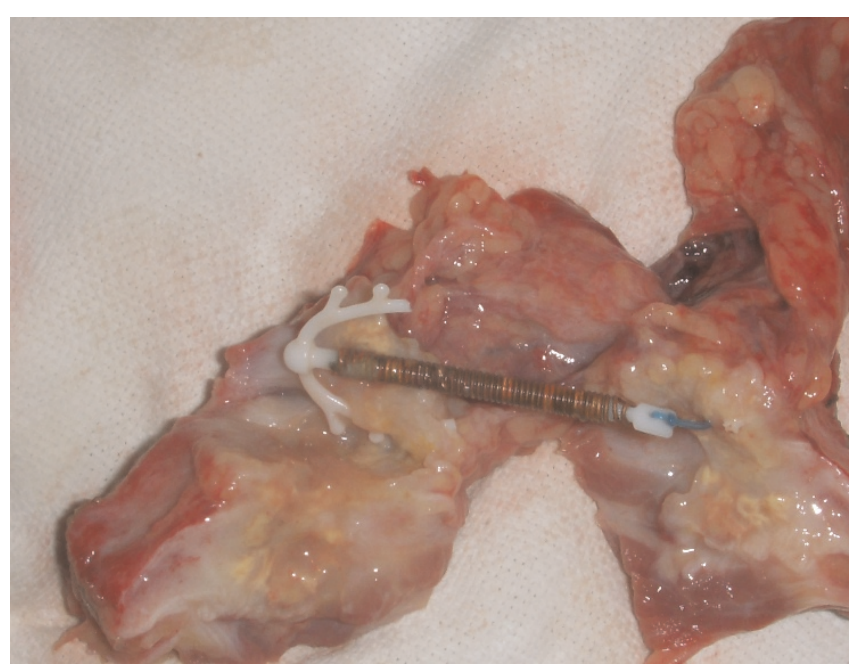

Figure I.

Copper- $T$ in the excised transected mass. of the anterior abdominal wall of the right iliac fossa, to which omentum was firmly adherent. The appendix was found to be normal and a wide-based Meckel's diverticulum was also present at 2 feet proximal to the ileo-caecal junction. Wide excision of the suspicious lesion was carried out with a clearance margin of $2 \mathrm{~cm}$ all round and the resultant fascio-muscular defect in the anterior abdominal wall was repaired with polypropylene mesh. The Meckel's diverticulum and the normal appendix were also excised.

The excised mass was bisected and, to our surprise, a copper-T IUCD was found embedded inside (Figure 1). The uterus was examined but there was no evidence of any perforation. The abdomen was closed and a tube drain was left in situ.

On cross-checking with the patient on the following day, she gave a history of copper-T insertion about 6 months previously.

The drain was removed after 48 hours, and the postoperative period was uneventful. The patient was discharged from the hospital on the 7th day after removal of stitches. She was asymptomatic at 1-month follow-up.

\section{Discussion}

Since their introduction in 1965, intrauterine contraceptive devices (IUCD) are commonly used as an effective, safe and economic method of long-term contraception. Translocation of an intrauterine contraceptive device to an extra-uterine site is an uncommon but potentially serious complication but this may remain asymptomatic or present with varying abdominal symptoms and signs, depending on the severity of involvement [2]. Migration to the urinary bladder is commonly reported [3]; however, a migrated copper- $\mathrm{T}$ has also been recovered from the rectum [4] and from the sigmoid colon [5-7]. Up to 2005, 15 cases of acute appendicitis induced by migrated IUCD have been reported [8]. To the best of our knowledge, this is the first report of IUCD migration to the anterior abdominal wall of the right iliac fossa.

In cases reported in the literature, the timing of extra-uterine presentation and the distant sites of translocation often raise the issue of whether iatrogenic uterine perforation or migration of the device was responsible. Primary iatrogenic uterine perforation usually occurs at the time of IUCD insertion but an IUCD may become embedded in the uterus and later be forced through the wall by spontaneous uterine contractions [9]. However, other possible translocatory mechanisms such as urinary bladder contractions, gut peristalsis and movement of peritoneal fluid may also play a significant role [10]. Factors contributing to the possibility of uterine perforation are inept insertion or 
positioning, fragility of the uterine wall due to recent birth, abortion or pregnancy in general. Chang and colleagues [8] also emphasized that the incidence is influenced by factors such as the timing of insertion, parity, type of IUD inserted, experience of the operator and position of the uterus. Increased risk of IUCD translocation has also been observed in lactating mothers [11].

A translocated IUCD induces a dense fibroblastic reaction [11] which is the usual cause of it occasionally not being detected on ultrasonography, as was the case in our patient, or routine laparoscopy $[2,12]$. Hence, plain X-ray of abdomen and pelvis, the classical routine investigation, but nowadays often forgotten in the heat of freely available ultrasounds and contrast enhanced computed tomography (CT) scans, appears to be more the reliable method, as has been emphasized by Katara and colleagues [2].

\section{Conclusions}

Uterine perforation and migration of IUCD usually passes unnoticed. Therefore, regular self-examination for "missing threads" supplemented with clinico-radiological controls in the follow-up after IUCD insertion can detect these migrations early. Easily available plain X-ray of abdomen and pelvis may be the simplest tool for early detection of a migrated IUCD and thereby avoid diagnostic difficulties and potentially serious complications.

\section{Consent}

Written informed consent was obtained from the patient for publication of this case report and any accompanying images. A copy of the written consent is available for review by the Editor-in-Chief of this journal.

\section{Competing interests}

The authors declare that they have no competing interests.

\section{Authors' contributions}

MMA was in charge of the overall care of the patient and researched the literature and prepared the manuscript, with RF and MFK involved in follow-up care and manuscript preparation. SH was solely responsible for anesthesia and postoperative recovery. Critical review and submission was carried out by SHH. All five authors read and approved the final manuscript.

\section{References}

I. Ohana E, Sheiner E, Leron E, Mazor M: Appendix perforation by an intrauterine contraceptive device. Eur J Obstet Gynecol Reprod Biol 2000, 88: |29-|3|.

2. Katara AN, Chandiramani VA, Pandya SM, Nair NS: Migration of intrauterine contraceptive device into the appendix. Indian J Surg 2004, 66: 179-180.

3. Sasidharan K, Chally R: Intravesical migration of Lippes loop with stone formation. Br J Urol 1998, 61:363-364.

4. Laxami M, Hemlata J, Rani LP: An unusual case of copper-T in the rectum. J Obstet Gynecol India 2005, 55:79-80.
5. Browning J], Bigrigg MA: Recovery of the intrauterine contraceptive device from the sigmoid colon. Three case reports. $\mathrm{Br}$ J Obstet Gynaecol 1988, 95:530-532.

6. Nceboz AES, Zakir HTA, Uyar Y, Ayar YH: Migration of an intrauterine contraceptive device to the sigmoid colon: a case report. Eur / Contracept Reprod Health Care 2003, 8:229-232.

7. Mansoor T, Aslam M, Rizwi SAA, Haseen MA: Copper-T causing perforation of sigmoid colon. Internet J Surg 2007, I 3(I).

8. Chang HM, Chen TW, Hsieh CB, Chen CJ, Yu JC, Liu YC, Shen KL, Chan DC: Intrauterine contraceptive device appendicitis: A case report. World J Gastroenterol 2005, II (34):54I4-54 I5.

9. Carson SA, Gatlin A, Mazur M: Appendiceal perforation by Copper-7 intrauterine contraceptive device. Am J Obstet Gynecol I98I, I 4 I (5):586-587.

10. Eke N, Okpani AOU: Extra-uterine translocated contraceptive device: $A$ presentation of five cases and revisit of the enigmatic issues of iatrogenic perforation and migration. Afr J Reprod Health 2003, 7(3): I I7-I 23.

II. Mittal S, Gupta I, Lata P, Mahajan U, Gupta AN: Management of translocated and incarcerated intrauterine contraceptive devices. Aust NZ J Obstet Gynaecol 1986, 26(3):232-234.

12. Kriplani A, Garg P, Sharma M, Agarwal N: Laparoscopic removal of extra-uterine IUCD using fluoroscopy guidance: a case report. J Gynecol Surg 2005, 2I(I):29-30.

\section{Do you have a case to share?}

Submit your case report today

- Rapid peer review

- Fast publication

- PubMed indexing

- Inclusion in Cases Database

\section{Any patient, any case, can teach us something}

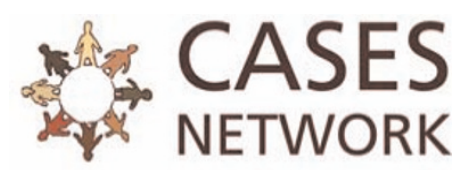

www.casesnetwork.com 